\title{
Patterns of multimorbidity and risk of severe SARS-CoV-2 infection: an observational study in the U.K.
}

Yogini V. Chudasama ${ }^{1 *} \mathbb{0}$, Francesco Zaccardi ' Clare L. Gillies ${ }^{1}$, Cameron Razieh², Thomas Yates², David E. Kloecker ${ }^{1}$, Alex V. Rowlands², Melanie J. Davies², Nazrul Islam³,4, Samuel Seidu' ${ }^{3}$, Nita G. Forouhi ${ }^{4 \dagger}$ and Kamlesh $\mathrm{Khunti}^{1+}$

\begin{abstract}
Background: Pre-existing comorbidities have been linked to SARS-CoV-2 infection but evidence is sparse on the importance and pattern of multimorbidity (2 or more conditions) and severity of infection indicated by hospitalisation or mortality. We aimed to use a multimorbidity index developed specifically for COVID-19 to investigate the association between multimorbidity and risk of severe SARS-CoV-2 infection.

Methods: We used data from the UK Biobank linked to laboratory confirmed test results for SARS-CoV-2 infection and mortality data from Public Health England between March 16 and July 26, 2020. By reviewing the current literature on COVID-19 we derived a multimorbidity index including: (1) angina; (2) asthma; (3) atrial fibrillation; (4) cancer; (5) chronic kidney disease; (6) chronic obstructive pulmonary disease; (7) diabetes mellitus; (8) heart failure; (9) hypertension; (10) myocardial infarction; (11) peripheral vascular disease; (12) stroke. Adjusted logistic regression models were used to assess the association between multimorbidity and risk of severe SARS-CoV-2 infection (hospitalisation/ death). Potential effect modifiers of the association were assessed: age, sex, ethnicity, deprivation, smoking status, body mass index, air pollution, 25-hydroxyvitamin D, cardiorespiratory fitness, high sensitivity C-reactive protein.

Results: Among 360,283 participants, the median age was 68 [range 48-85] years, most were White (94.5\%), and 1706 had severe SARS-CoV-2 infection. The prevalence of multimorbidity was more than double in those with severe SARS-CoV-2 infection (25\%) compared to those without (11\%), and clusters of several multimorbidities were more common in those with severe SARS-CoV-2 infection. The most common clusters with severe SARS-CoV-2 infection were stroke with hypertension (79\% of those with stroke had hypertension); diabetes and hypertension (72\%); and chronic kidney disease and hypertension (68\%). Multimorbidity was independently associated with a greater risk of severe SARS-CoV-2 infection (adjusted odds ratio 1.91 [95\% confidence interval 1.70,2.15] compared to no multimorbidity). The risk remained consistent across potential effect modifiers, except for greater risk among older age. The highest risk of severe infection was strongly evidenced in those with CKD and diabetes (4.93 [95\% Cl 3.36, 7.22]).
\end{abstract}

Conclusion: The multimorbidity index may help identify individuals at higher risk for severe COVID-19 outcomes and provide guidance for tailoring effective treatment.

\footnotetext{
*Correspondence: yc244@leicester.ac.uk

${ }^{\dagger}$ Nita G. Forouhi and Kamlesh Khunti are joint senior authors

${ }^{1}$ Leicester Real World Evidence Unit, Diabetes Research Centre, Leicester

General Hospital, University of Leicester, Leicester, UK

Full list of author information is available at the end of the article
} 
Keywords: Multimorbidity, Pattern, SARS-CoV-2 infection, COVID-19, Hospitalisation, Mortality, Risk factors

\section{Background}

The novel coronavirus SARS-CoV-2 has led to a global pandemic of a complex, pneumonia-related illness (COVID-19). As of 29th September 2020, there have been 33,749,599 confirmed cases of COVID-19 and $1,009,829$ deaths reported worldwide [1]. Many countries are still facing a devastating rise in the number of cases and deaths, whilst some are facing a second wave such as France, Spain, UK, with significant impacts on their healthcare systems. The risk of SARS-CoV-2 infection is based on the person-to-person transmission rate at which the virus is circulating in the community $[2,3]$. Yet, while some individuals recover from the SARS-CoV-2 infection, others develop severe illness which may require intensive care in hospital or result in mortality.

Several potential factors have been postulated or associated with the increased risk of transmission and development of severe SARS-CoV-2 infection, such as age, sex, ethnicity, body mass index (BMI), smoking, deprivation, pre-existing comorbidities (particularly diabetes, hypertension and cardiovascular disease), cardiorespiratory fitness, 25-hydroxyvitamin D level, air pollution and inflammation [4]. However, individuals with pre-existing health conditions have been found to have the highest risk of developing severe SARS-CoV-2 infection, as shown in a number of studies from early research based in Wuhan [5] to recent studies across the globe [6-8]. Nevertheless, the majority of research to date has focused on pre-existing single chronic conditions [9], yet individuals often have multiple chronic conditions, known as multimorbidity. In view of the rapidly increasing prevalence of severe SARS-CoV-2 infection across the globe, clarifying the pattern and impact of multimorbidity (defined as 2 or more conditions) on the risk of severe SARS-CoV-2 infection has important clinical and public health implications which are not captured by a focus on single diseases [10].

There are a number of methods to define multimorbidity, which include both simple measures such as summing up the diseases and more complex measures using a weighted score such as the Charles Comorbidity Index. A previous study in UK Biobank examined the pattern of the most prevalent clusters of multimorbidity in those infected with SARS-CoV-2, using 36 different conditions to define multimorbidity [11], though this masked the most relevant conditions related to SARSCoV-2 [12]. Moreover, three studies which assessed the risk of multimorbidity and COVID-19 had also used a broad range of conditions (ranging from 19 to 43 conditions) [13-15]. In contrast, the available evidence indicates that certain health conditions are associated with a much greater risk of severe SARS-CoV-2 infection linked with hospitalisation or mortality $[5,16]$.

To help clarify the evidence, in this study we aimed to firstly develop a multimorbidity index of the most commonly reported chronic conditions from the current literature for severe SARS-CoV-2 infection. The index was then used to investigate the patterns and association between multimorbidity and risk of severe SARS-CoV-2 infection and we further tested whether this association was modified by other potential risk factors.

\section{Methods}

This study is reported as per the Strengthening the Reporting of Observational Studies in Epidemiology (STROBE) guidelines (Additional File 1: Checklist S1) and following a pre-specified protocol (Application Number 36371) [17].

\section{Study population}

UK Biobank included half a million middle-aged adults recruited from 22 sites across England, Wales and Scotland with baseline measures collected between 2006 and 2010, from an in-person baseline interview at the UK Biobank centre (http://biobank.ctsu.ox.ac.uk/crystal/ search.cgi). Written informed consent was obtained prior to data collection; UK Biobank was approved by the NHS National Research Ethics Service (16/NW/0274; ethics approval for UK Biobank studies) [18].

SARS-CoV-2 laboratory confirmed test results and death data for all-cause mortality from Public Health England were linked to the UK Biobank database [19]. Data were available between March 16 and July 26, 2020, restricted to those tested in a hospital (pillar 1), since this can be regarded as a proxy for hospitalisations for severe cases of the disease as suggested by the linkage methodology [19]. Our analytical sample included study participants from England as testing was available and linked, we therefore excluded study members from Scotland and Wales, participants who had an outpatient test positive for COVID-19 (pillar 2) as the outcome for SARS-CoV-2 infection was uncertain (i.e. recovered, hospitalised at a later stage, or died), those who died before March 16, 2020, and those with missing data (Additional file 1: Fig. S1). 


\section{Multimorbidity index}

To use prior evidence on comorbidities that cluster together and that may act in combination to affect the risk of COVID-19, we carried out a literature search to identify the most common pre-existing comorbidities in patients with severe SARS-CoV-2 infection (Additional file 1: Table S1). The studies from the literature search were found to be very heterogeneous, therefore it was not possible to do a quantitative meta-analysis of pooled results $[5-8,20-29]$. From a semi-quantitative perspective, we selected the most common conditions to create a multimorbidity index for severe COVID-19 including the following conditions: (1) angina; (2) asthma; (3) atrial fibrillation; (4) cancer; (5) chronic kidney disease (CKD); (6) chronic obstructive pulmonary disease (COPD); (7) diabetes mellitus; (8) heart failure; (9) hypertension; (10) myocardial infarction; (11) peripheral vascular disease; and (12) stroke. Data on these comorbidities were available in UK Biobank together with the clinical marker of CKD, the estimated Glomerular Filtration Rate (eGFR) value less than $60 \mathrm{~mL} / \mathrm{min} / 1.73 \mathrm{~m}^{2}$. Those with two or more of these conditions clustering together were defined as having multimorbidity and the remainder without multimorbidity.

\section{Outcome measure}

The outcome was severe SARS-CoV-2 infection, defined as admission to hospital with a positive SARS-CoV-2 infection test result or death occurring within the study period of March 16 to July 26 July, 2020. The comparison group without severe SARS-CoV-2 infection included participants with negative test result for SARS-CoV-2 either in a hospital setting or as outpatient, or having not been tested. Participants who received a positive test outside of a hospital setting were excluded from this analysis, as their outcome was uncertain (i.e. recovered, hospitalised at a later stage, or died).

\section{Effect modifiers}

The association between multimorbidity and severe SARS-CoV-2 infection was assessed across levels of ten prespecified potential effect modifiers based on the literature [4], including: (1) age at test (grouped as $<60$ years or $\geq 60$ years; (2) sex; (3) ethnicity (self-reported and grouped as white or non-white); (4) Townsend deprivation index (used to measure socio-economic status, which combines census data on housing, employment, and social class based on the postal code of participants; a higher Townsend score equates to higher levels of socioeconomic deprivation and participants were split in two groups based on the sample median: least or most deprived); (5) body mass index (BMI), following the exclusion of individuals in the underweight $[<18.5 \mathrm{~kg} /$ $\mathrm{m}^{2}$ ] group $(\mathrm{n}=2093)$, since $<1 \%$ of the participants were categorised as underweight; the remaining participants were grouped as: normal weight $\left[18.5-24.9 \mathrm{~kg} / \mathrm{m}^{2}\right]$, overweight $\left[25-29.9 \mathrm{~kg} / \mathrm{m}^{2}\right]$, or obese $\left[\geq 30 \mathrm{~kg} / \mathrm{m}^{2}\right]$ ); (6) smoking (current smoker, previous smoker or never smoked at the time of assessment); (7) the annual average air pollution, measured by the concentrations of nitrogen dioxide $\left(\mathrm{NO}_{2}\right)$; data for the earliest year when the participant attended the assessment centre was used. We used the World Health Organization (WHO) guideline threshold of $40 \mu \mathrm{g} / \mathrm{m}^{3}$ (annual mean) which was set as the level for protecting the public from the health effects of gaseous pollutants, with values $\geq 40 \mu \mathrm{g} / \mathrm{m}^{3}$ assigned as high [30]; (8) serum 25-hydroxyvitamin D (25(OH) D) concentration, a measure of vitamin D status, was measured at a central laboratory and further details of biochemical assays are provided elsewhere (http://bioba nk.ctsu.ox.ac.uk/crystal/refer.cgi?id=5636). In line with the recommendation to maintain sufficient amounts of blood vitamin D levels at $\geq 25 \mathrm{nmol} / \mathrm{L}$ in the vast majority (97.5\%) of the population in the UK [31], participants were grouped as clinically deficient $(<25 \mathrm{nmol} / \mathrm{L})$, or sufficient ( $\geq 25 \mathrm{nmol} / \mathrm{L}$ ) based on their $25(\mathrm{OH}) \mathrm{D}$ level; (9) cardiorespiratory fitness, defined by self-reported walking pace (slow walking pace, or steady-brisk walking pace [32]); and (10) the inflammatory marker high sensitivity $\mathrm{C}$-reactive protein $(\mathrm{CRP}$, normal $[<3 \mathrm{mg} / \mathrm{L}]$ or high $[\geq 3 \mathrm{mg} / \mathrm{L}])$ based on clinical recommendations [33].

\section{Statistical analyses}

We examined the baseline descriptive characteristics of the UK Biobank cohort among participants with and without severe SARS-CoV-2 infection. The age at the time of SARS-CoV-2 test was calculated using the baseline date and the test date. Continuous variables were presented as means and standard deviations, and the categorical variables presented as counts and percentages. Complete case framework was used for all analyses. A heat map was created to illustrate the pattern of the most common clusters of multimorbidity by severe SARSCoV-2 infection and the remaining cohort. The proportion and date of those with severe SARS-CoV-2 infection by multimorbidity was also illustrated to understand the pattern over the time of the pandemic.

The association between multimorbidity and severe SARS-CoV-2 infection was estimated using logistic regression models. The odds ratio (OR) and $95 \%$ confidence intervals $(\mathrm{CI})$ were reported comparing multimorbidity versus no multimorbidity (reference). Models were adjusted for age at test, sex, ethnicity, deprivation, smoking status, BMI, air pollution, serum 25-hydroxyvitamin $\mathrm{D}$, cardiorespiratory fitness, serum C-reactive protein, 
season at blood draw [categorised by the months: spring (March, April, May), summer (June, July, August), autumn (September, October, November), and winter (December, January, February)], and regular intake of vitamin D supplement (yes or no). Stratified analyses were used for the ten potential effect modifiers, and we tested for interaction effects using a Likelihood ratio test and the related $\mathrm{P}$-value.

In sensitivity analyses to assess the robustness of our results, we repeated the stratified analyses using 3 or more comorbid conditions. Moreover, we further stratified the analyses using four 25-hydroxyvitamin D levels $(<25,25-50,50-75,>75 \mathrm{nmol} / \mathrm{L})$ and also included a more recent testing phase for 25-hydroxyvitamin D levels; and we used the air pollution level markers $\mathrm{NO}_{2}$ and PM 2.5 at the last recorded measure. Since the other potential confounders were measured only once prior to the pandemic, we additionally accounted for the time between the baseline data collection and the date of hospitalisation of SARS-CoV-2, mortality, or censoring. In addition, the adjustment for cardiorespiratory fitness and level of serum C-reactive protein could be seen as a consequence of co-morbidities, therefore we assessed the results with these potential mediators removed. A P-value less than 0.05 was considered statistically significant. All analyses were performed in Stata version 16.0.

\section{Results}

\section{Participant characteristics}

In 360,283 UK Biobank participants, 1706 (0.5\%) had severe SARS-CoV-2 infection. The median [range] age was 68 [48-85] years and most participants were White (94.5\%). Compared with participants without severe SARS-CoV-2 infection, those with severe infection were older ( $\geq 60$ years: $86 \%$ vs. $77 \%$ ), mainly men ( $58 \%$ vs. $46 \%)$, more likely to live in deprived areas (60\% vs. $50 \%)$, with higher mean BMI $\left(28.6 \mathrm{~kg} / \mathrm{m}^{2}\right.$ vs $\left.27.4 \mathrm{~kg} / \mathrm{m}^{2}\right)$, were current or previous smokers, had vitamin D deficiency (16\% vs.12\%), and more frequently reported a slow walking pace (17\% vs. $7 \%)$; Table 1.

\section{Pattern of multimorbidity}

The most prevalent pre-existing multimorbidity index conditions in those with severe SARS-CoV-2 infection were hypertension (39.6\%), asthma (13.3\%), diabetes (10.0\%), cancer $(9.9 \%)$, and angina (6.5\%; Table 2). There was a higher prevalence of hypertension ( $39.6 \%$ vs. $25.3 \%$ ) and diabetes $(10 \%$ vs. $3.9 \%)$ in individuals with severe SARS-CoV-2 infection compared to those without. The prevalence of multimorbidity (2 or more conditions) was over double in those with severe SARS-CoV-2 infection (25.3\%) compared to those without severe SARS-CoV-2 infection (11.6\%; Table 2), where the most common clusters were found in those with both stroke and hypertension (79\% of those with stroke had hypertension), diabetes and hypertension (72\% of those with diabetes), CKD and hypertension (68\% of those with CKD), angina and hypertension (67\% of those with angina; Fig. 1). The trend of severe SARS-CoV-2 infection cases in this study peaked at the end of March and beginning of April 2020, and those with multimorbidity had a consistently higher proportion of cases during the period compared to those without multimorbidity; Fig. 2.

\section{Risk of severe SARS-CoV-2 infection}

The risk of severe SARS-CoV-2 infection increased with the number of pre-existing multimorbidity index conditions, illustrated by the higher odds ratios across the number of increasing conditions (Table 3 ). The overall combined effect in those with multimorbidity ( 2 or more conditions) was associated with around 2.6 times greater risk of severe SARS-CoV-2 infection compared to those without multimorbidity (crude OR 2.56 [2.32, 2.89]); and, in the adjusted model, nearly two times greater (adjusted OR 1.91 [1.70, 2.15]), Table 3. The odds ratios remained generally consistent with the overall associations when estimated across strata of effect modifiers (Fig. 3). The association was stronger in those over the age of 60 years compared to under 60 years, and slightly stronger in men compared to women (Fig. 3). It was also apparent that the risk differed across the most common clusters of multimorbidity (Table 4) with clusters containing asthma \& hypertension having the lowest risk (adjusted OR 1.29 $[1.03,1.60])$ and those containing CKD \& diabetes having the highest risk (adjusted OR 4.93 [3.36, 7.22]).

Results were consistent in sensitivity analyses considering the following: (1) when examining the association between the multimorbidity index using 3 or more comorbid conditions and the risk of severe SARS-CoV-2 infection, however the interaction effect between men and women was non-significant; (2) when assessing the $25 \mathrm{OH}$-vitamin D levels using further stratifications; (3) and at a later phase; (4) when using the last recorded air pollution $\mathrm{NO}_{2}$ and PM 2.5 levels; (4) after adjusting for the time difference; and (5) when removing the confounders cardiorespiratory fitness and level of serum C-reactive protein (see Additional file 1: Tables S2, S3, and S4).

\section{Discussion}

Our review of the published literature indicated that the most commonly reported chronic conditions for severe SARS-CoV-2 infection were cardiometabolic diseases. Data from the UK Biobank study using the multimorbidity index conditions showed that $25 \%$ of those with severe SARS-CoV-2 infection had multimorbidity (2 or more 
Table 1 Characteristics of UK Biobank participants

\begin{tabular}{|c|c|c|c|}
\hline Characteristics & $\begin{array}{l}\text { With severe SARS-CoV- } 2 \text { infection }^{a} \\
N=1706(0.5 \%)\end{array}$ & $\begin{array}{l}\text { Without severe SARS-CoV-2 } \\
\text { infection }^{b} \\
\mathrm{~N}=358,577(99.5 \%)\end{array}$ & $P$ value \\
\hline Current age, mean [SD] & $71.0[8.0]$ & $67.2[8.1]$ & $<0.001$ \\
\hline$<60$ years & $248(14.5)$ & $83,021(23.2)$ & \\
\hline$\geq 60$ years & $1458(85.5)$ & $275,556(76.9)$ & $<0.001$ \\
\hline \multicolumn{4}{|l|}{ Sex } \\
\hline Women & $725(42.5)$ & $194,846(54.3)$ & \\
\hline Men & $981(57.5)$ & $163,731(45.7)$ & $<0.001$ \\
\hline \multicolumn{4}{|l|}{ Ethnicity } \\
\hline White & $1567(91.9)$ & $339,052(94.6)$ & \\
\hline Non-white & $139(8.2)$ & $19,525(5.5)$ & $<0.001$ \\
\hline Deprivation index, mean [SD] & $-0.6[3.4]$ & $-1.4[3.0]$ & \\
\hline Least deprived & $699(41.0)$ & $179,448(50.0)$ & \\
\hline Most deprived & 1007 (59.0) & $179,129(50.0)$ & $<0.001$ \\
\hline Body mass index $\left(\mathrm{kg} / \mathrm{m}^{2}\right)$, mean [SD] & $28.6[5.3]$ & $27.4[4.7]$ & \\
\hline Normal $\left(18.5-24.9 \mathrm{~kg} / \mathrm{m}^{2}\right)$ & $426(25.0)$ & $120,338(33.6)$ & \\
\hline Overweight $(25-29.9$ kg/m²) & $729(42.7)$ & $153,185(42.7)$ & $<0.001$ \\
\hline Obese $\left(\geq 30 \mathrm{~kg} / \mathrm{m}^{2}\right)$ & $551(32.3)$ & $85,054(23.7)$ & \\
\hline \multicolumn{4}{|l|}{ Smoking status } \\
\hline Never & $772(45.3)$ & $199,897(55.8)$ & \\
\hline Previous & $699(41.0)$ & $124,183(34.6)$ & \\
\hline Current & $235(13.8)$ & $34,497(9.6)$ & $<0.001$ \\
\hline Air pollution $\mathrm{NO}_{2}\left(\mu \mathrm{g} / \mathrm{m}^{3}\right)$ mean $[\mathrm{SD}]$ & $29.5[8.8]$ & $28.0[8.6]$ & \\
\hline Low/moderate level $(<40$ mg/m³) & $1547(90.7)$ & $333,831(93.1)$ & \\
\hline High level $\left(\geq 40 \mu \mathrm{g} / \mathrm{m}^{3}\right)$ & $159(9.3)$ & $24,746(6.9)$ & $<0.001$ \\
\hline 25(OH) D (nmol/L), mean [SD] & $47.3[21.6]$ & $49.6[21.0]$ & \\
\hline Severe deficiency (<25 nmol/L) & $278(16.3)$ & $43,280(12.1)$ & \\
\hline Sufficient $(\geq 25 \mathrm{nmol} / \mathrm{L})$ & $1428(83.7)$ & $315,297(87.9)$ & $<0.001$ \\
\hline Regular vitamin D supplements intake & $81(4.8)$ & $14,295(4.0)$ & 0.109 \\
\hline \multicolumn{4}{|l|}{ Season of blood draw } \\
\hline Winter & $328(19.2)$ & $66,579(18.6)$ & \\
\hline Spring & $491(28.8)$ & $99,791(27.8)$ & \\
\hline Summer & $490(28.7)$ & $104,251(29.1)$ & \\
\hline Autumn & $397(23.3)$ & $87,956(24.5)$ & 0.548 \\
\hline \multicolumn{4}{|l|}{ Walking pace } \\
\hline Slow pace & $290(17.0)$ & $25,279(7.1)$ & \\
\hline Steady/brisk pace & $1416(83.0)$ & $333,298(93.0)$ & $<0.001$ \\
\hline C-reactive protein (mg/L), mean [SD] & $3.3[5.4]$ & $2.5[4.1]$ & \\
\hline Normal $(<3$ mg/L) & $1213(71.1)$ & $281,507(78.5)$ & \\
\hline High ( $\geq 3$ mg/L) & $493(28.9)$ & $77,070(21.5)$ & $<0.001$ \\
\hline
\end{tabular}

$\mathrm{N}$ (\%) presented, unless stated otherwise. Deprivation defined by Townsend deprivation index. P-values calculated by t-test or Chi squared test $\mathrm{NO}_{2}$ nitrogen dioxide

a Severe SARS-CoV-2 infection included those with a positive test result for SARS-CoV-2 with hospitalisation, or those who died during the study period (16 March to 26 July, 2020)

${ }^{\mathrm{b}}$ Those without severe SARS-CoV-2 infection included those who had not been tested, or who had tested negative for SARS-CoV-2

conditions). The major clusters were stroke and hypertension; diabetes and hypertension; CKD and hypertension; and angina and hypertension. Over the study period the risk of severe SARS-CoV-2 infection was consistently higher for those with multimorbidity compared to those without multimorbidity. Moreover, our results highlight that the greater risk of severe infection was consistent with that of the overall effect of multimorbidity when 
Table 2 Multimorbidity index conditions among participants in the UK Biobank Study

\begin{tabular}{|c|c|c|c|}
\hline Multimorbidity index conditions & $\begin{array}{l}\text { With severe SARS-CoV-2 } \\
\text { infection }^{\mathrm{a}} \\
\mathrm{N}=1706(0.5 \%)\end{array}$ & $\begin{array}{l}\text { Without severe SARS-CoV-2 } \\
\text { infection } \\
\mathrm{N}=358,577(99.5 \%)\end{array}$ & $P$ value \\
\hline Hypertension & $676(39.6)$ & $90,747(25.3)$ & $<0.001$ \\
\hline Asthma & $226(13.3)$ & $41,382(11.5)$ & 0.028 \\
\hline Diabetes & $170(10.0)$ & $14,079(3.9)$ & $<0.001$ \\
\hline Cancer & $179(10.5)$ & $27,108(7.6)$ & $<0.001$ \\
\hline Angina & $111(6.5)$ & $10,037(2.8)$ & $<0.001$ \\
\hline Myocardial infarction & $102(6.0)$ & $7096(2.0)$ & $<0.001$ \\
\hline Chronic kidney disease & $93(5.5)$ & $7188(2.0)$ & $<0.001$ \\
\hline Stroke & $47(2.8)$ & $3957(1.1)$ & $<0.001$ \\
\hline Atrial fibrillation & $26(1.5)$ & $2508(0.7)$ & $<0.001$ \\
\hline Chronic obstructive pulmonary disease & $14(0.8)$ & $929(0.3)$ & $<0.001$ \\
\hline Heart failure & $5(0.3)$ & $146(0.04)$ & $<0.001$ \\
\hline Peripheral vascular disease & $4(0.2)$ & $595(0.2)$ & 0.488 \\
\hline \multicolumn{4}{|l|}{ Number of multimorbidity index conditions } \\
\hline None & $683(40.0)$ & $206,012(57.5)$ & \\
\hline One & $592(34.7)$ & $111,153(31.0)$ & \\
\hline Two & $293(17.2)$ & $32,059(8.9)$ & \\
\hline Three & $96(5.6)$ & $7341(2.1)$ & \\
\hline Four & $27(1.6)$ & $1646(0.5)$ & \\
\hline Five & $12(0.7)$ & $311(0.1)$ & \\
\hline Six & $2(0.1)$ & $47(0.01)$ & \\
\hline Seven & $1(0.1)$ & $7(0.0)$ & \\
\hline Eight & $0(0.0)$ & $1(0.0)$ & $<0.001$ \\
\hline
\end{tabular}

P-values calculated by chi squared test

a Severe SARS-CoV-2 infection included those with a positive test result for SARS-CoV- 2 with hospitalisation, or those who died during the study period (16 March to 26 July, 2020)

${ }^{b}$ Those without severe SARS-CoV-2 infection included those who had not been tested, or who had tested negative for SARS-CoV-2

stratifying by the potential effect modifiers. The only exception was when stratifying by age, with a greater magnitude of association between multimorbidity and severe infection in those over 60 years compared to those under 60 years. Although a statistically significant difference was also found by sex $(P=0.020)$, the estimated odds ratios were similar in both men and women, indicating less clinical importance for this modifier. The association between the most common co-occurring multimorbidity clusters and risk of severe SARS-CoV-2 infection was strongly evidenced in those with pre-existing CKD and diabetes.

To our knowledge, this is the first study to develop a multimorbidity index using the most common conditions from the current COVID-19 literature. There have been three previously published studies that assessed the association between multimorbidity and the risk of SARS$\mathrm{CoV}-2$ infection. The first was an online questionnaire study by 26 hospitals across Italy undertaken in March 9 to April 9, 2020, with a total of 1591 participants admitted with SARS-CoV-2 infection (188 non-survivors, 1403 survivors) [14]. The study used the Charles Comorbidity Index (CCI) which takes into account age and 19 conditions in an algorithm to predict 10 -year mortality. The authors found an exponential increase in the odds by each point of the CCI score [14]. Our multimorbidity index found similar results since the risk of severe SARS-CoV-2 infection increased with the number of pre-existing multimorbidity index conditions. Two studies were based in UK Biobank, however the study design and outcomes differed which resulted in inconsistent findings. One study in 4510 UK Biobank participants between 16 March to 1 June, 2020 (1326 tested positive for COVID-19 and 3184 tested negative) found in adjusted analyses that the number of comorbidity groupings $(0-1,2,3, \geq 4)$ was not associated with the risk of testing positive for COVID19 when compared to those who tested negative: the OR was 1.0 [ $95 \%$ CI $0.8,1.2]$ comparing those with 2 conditions vs. $0-1$ conditions; or only slightly increased when compared to the remaining population (OR $1.3[1.1,1.5]$ ) [13]. However, this study used 43 different conditions to define multimorbidity, and compared only those testing 


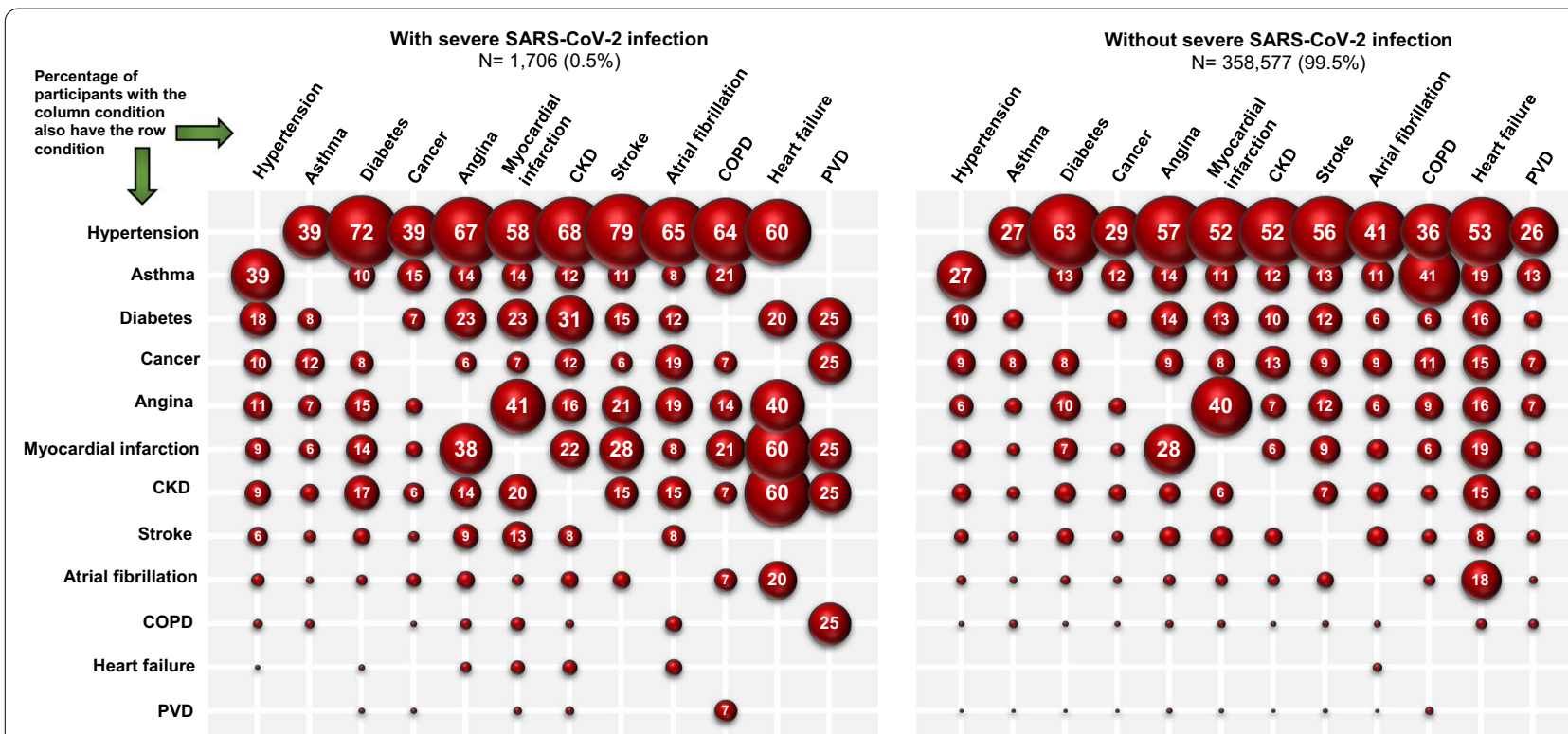

Fig. 1 Two most common pre-existing multimorbidity index conditions in UK Biobank participants with and without severe SARS-CoV-2 infection (March 16 to July 26, 2020). CKD chronic kidney disease; COPD chronic obstructive pulmonary disease; PVD peripheral vascular disease

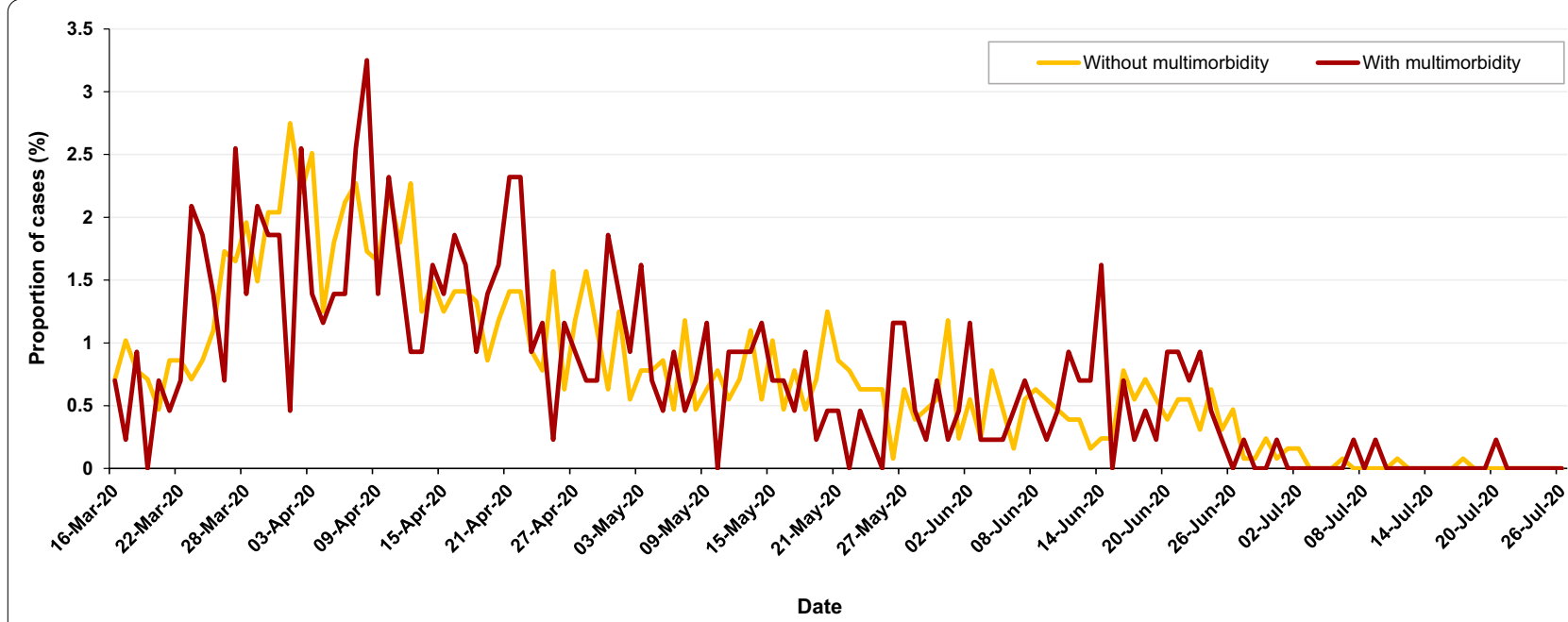

Fig. 2 Comparing the prevalence and date of those diagnosed with severe SARS-CoV-2 infection (hospitalisation or death, $N=1706$ ) by multimorbidity index ( $\geq 2$ conditions) in the UK Biobank. Without multimorbidity $(n=1275)$; with multimorbidity $(n=431)$. Multimorbidity index conditions include: angina, asthma, atrial fibrillation, cancer, chronic kidney disease, chronic obstructive pulmonary disease, diabetes mellitus, heart failure, hypertension, myocardial infarction, peripheral vascular disease, and stroke

positive compared to negative. The other UK Biobank study, between 16 March and 18 May, 2020 (1324 tested positive for COVID-19 and 426,875 remaining cohort), used Poisson regression analyses reporting that those with multimorbidity (defined using 43 conditions) had a 48\% higher risk for a positive test for COVID-19 (RR 1.48 [95\% CI 1.28, 1.71], $\mathrm{P}<0.01)$. Although this study also reported that those with cardiometabolic multimorbidity, compared with those without, had a 77\% higher risk for a positive test (RR $1.77[1.46,2.15] \mathrm{P}<0.01)$ [15], the outcome was based on testing positive in any setting (hospital or outpatient, pillar 1 and 2), which may introduce testing bias within UK Biobank. A recent article illustrated how the same dataset can produce apparently paradoxical findings for the same outcome [34]. For this reason, our study examined harder outcomes for those 
Table 3 Association between the number of multimorbidity index conditions and the risk of severe SARS-CoV-2 infection: UK Biobank ( $N=360,283)$

\begin{tabular}{lll}
\hline $\begin{array}{l}\text { Risk of severe SARS- } \\
\text { CoV-2 infection } \\
\text { (hospitalisation or } \\
\text { death) }\end{array}$ & Crude OR $\mathbf{( 9 5 \% ~ C l )}$ & Adjusted OR (95\% CI) \\
\hline$\geq 1$ vs. 0 (reference) & $2.02(1.84,2.23)$ & $1.65(1.49,1.82)$ \\
$\geq 2$ vs. $<2$ (reference) & $2.59(2.32,2.89)$ & $1.91(1.70,2.15)$ \\
$\geq 3$ vs. $<3$ (reference) & $3.29(2.76,3.92)$ & $2.02(1.68,2.43)$ \\
$\geq 4$ vs. $<4$ (reference) & $4.47(3.28,6.09)$ & $2.25(1.64,3.09)$
\end{tabular}

Multimorbidity index conditions include: angina, asthma, atrial fibrillation, cancer, chronic kidney disease, chronic obstructive pulmonary disease, diabetes mellitus, heart failure, hypertension, myocardial infarction, peripheral vascular disease, and stroke

Models adjusted for age at test, sex, ethnicity, deprivation, smoking status, body mass index, air pollution, 25 -hydroxyvitamin $\mathrm{D}$, cardiorespiratory fitness, C-reactive protein, season at blood draw, and regular intake of vitamin D supplement

OR odds ratio, $\mathrm{Cl}$ confidence interval

with severe SARS-CoV-2 infection; hospitalisation or death during the pandemic compared to the remaining cohort. Moreover, the three studies to date have only presented the number of conditions but have not examined the patterns of multimorbidity, i.e. the type of the two most common co-occurring conditions found in those with severe SARS-CoV-2 infection, nor the rate of severe infection or mortality in people with multimorbidity over the period of the pandemic.

Hypertension was found to be the most prevalent condition (40\%) in those with severe SARS-CoV-2 infection, as in a number of previously published studies. Yet, when examined by clusters, we found that hypertension mainly co-existed with other conditions (i.e. diabetes, stroke, CKD). Thus, certain clusters of the multimorbidity index are more frequent than other clusters. When examining the association between the most common co-occurring multimorbidity clusters and the risk of severe SARS-CoV-2 infection, those with pre-existing CKD and diabetes showed the strongest association. For this reason, we believe that the classification of those with pre-existing multimorbidity index conditions is crucial to provide prognostic information to tailor effective treatment and prevent severe outcomes in common chronic conditions. Individuals with pre-existing multimorbidity conditions may have potential organ dysfunctions, which are further accelerated by the local and systemic effects related to SARS-CoV-2 infection. Previous studies have investigated the possible pathophysiological mechanisms linking this infection to severe COVID-19 syndrome, including increased inflammation, decreased immune response, heart failure, renal failure, and potentially multi-system organ failure and death $[35$,
36]. Further research is however required to understand the underlying biological mechanisms involved between these multimorbidity clusters and the increased risk of severe COVID-19 and its long-term sequelae (i.e., "long COVID"), particularly the effects on the immune system and frailty.

Multimorbidity has been associated with premature mortality, lower quality of life, polypharmacy, frailty, and fragmented care [10]. However, the current pandemic has placed additional risks on individuals with multimorbidity. A global survey evaluating the impact of COVID-19 on routine care for chronic diseases during 31 March to 23 April 2020 found that most healthcare professionals $(67 \%)$ identified moderate or severe effects on their patients due to changes in healthcare services since the outbreak; and $80 \%$ reported the mental health of their patients worsened during COVID-19 [37]. Moreover, many low and middle-income countries have overstretched healthcare systems and the pandemic has further overwhelmed them.

A key strength of this study is the overall large sample size which allowed us to investigate whether the associations were heterogeneous across clinically relevant effect modifiers. We employed a comprehensive list of effect modifiers, extending past research [4], and enabling a greater understanding of the association between multimorbidity and severe SARS-CoV-2 infection. Moreover, we performed a literature review to derive an evidence based COVID-19 relevant multimorbidity index which can be applied to other populations [5-8, 20-29]. This study has some limitations. Issues of the low response rate $(\sim 5 \%)$ and selection bias, such as slightly higher representation of participants from affluent groups may suggest that the UK Biobank sample is not well representative of the UK population, and have been discussed previously [38]. The characteristics we examined were recorded at recruitment, representing data collected in the past at study baseline, but we conducted additional sensitivity analyses using the follow-up data for 25-hydroxyvitamin $\mathrm{D}$ levels and the last recorded air pollution $\left(\mathrm{NO}_{2}\right)$ data, showing consistent results over time. In further sensitivity analyses, we additionally adjusted the model for the time in the study, and found the results remained consistent. Factors such as cardiorespiratory fitness or C-reactive protein could possibly be a consequence of poor health from multimorbidity instead of cause, for this reason we removed these from the model, and the results remained the same. The data on multimorbidity were appraised only at baseline but there may have been a change in the number of multimorbidity index conditions since recruitment which were not accounted for and may lead to under or 


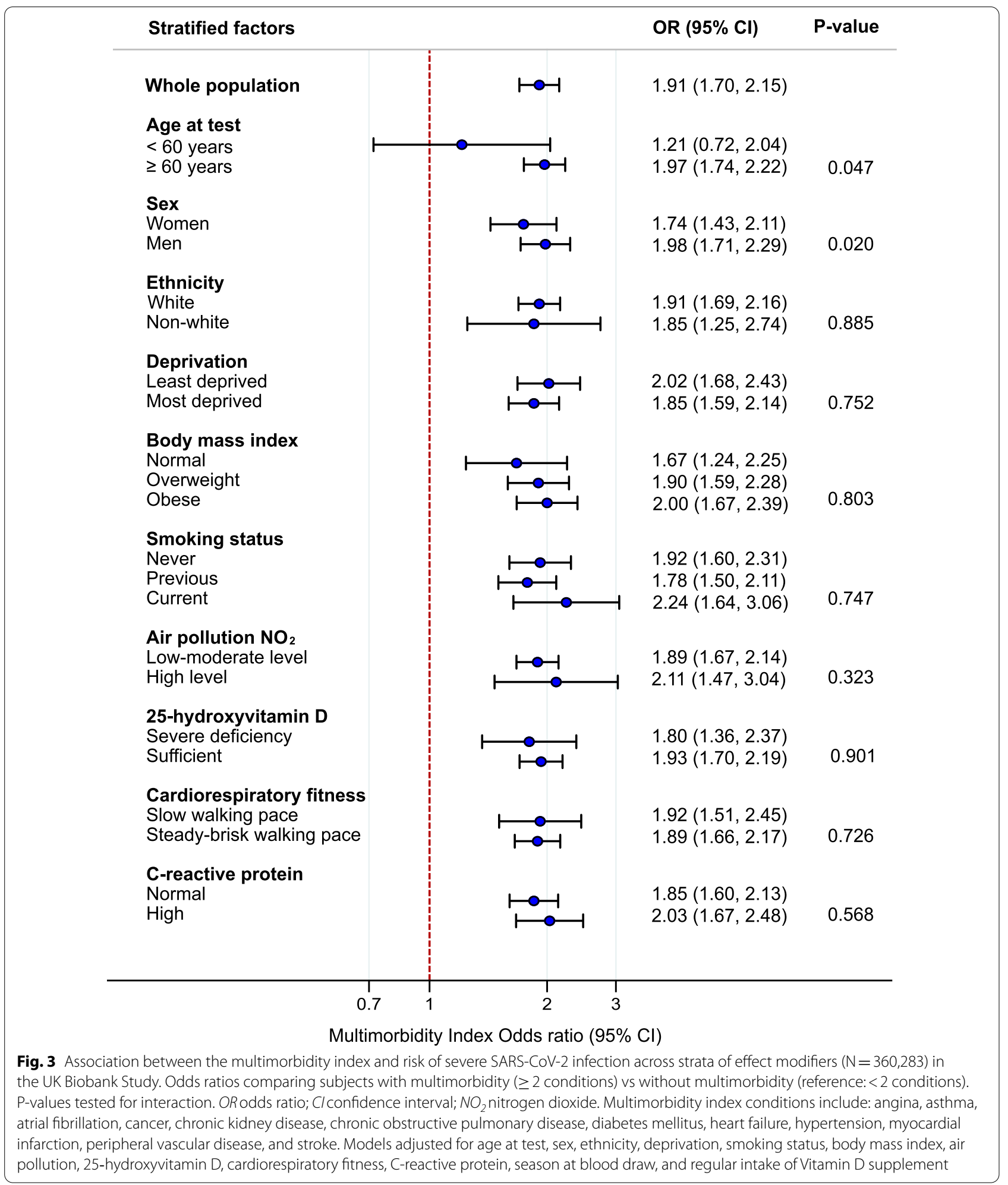

overestimation of effect. We were unable to assess the severity of disease conditions but our new multimorbidity index definition included conditions that were most relevant to COVID-19. Another limitation is the lack of detail of clinical severity of those with severe SARS-CoV-2 infection: while we have accounted for 
Table 4 Association between the most common co-occurring multimorbidity index conditions and the risk of severe SARSCoV-2 infection: UK Biobank $(N=360,283)$

\begin{tabular}{ll}
\hline $\begin{array}{l}\text { Risk of severe SARS-CoV-2 infection } \\
\text { (hospitalisation or death) }\end{array}$ & Adjusted OR (95\% C) \\
\hline CKD \& diabetes $(n=713)$ & $4.93(3.36,7.22)$ \\
Heart failure \& hypertension $(n=81)$ & $4.47(1.40,14.29)$ \\
COPD \& hypertension $(n=343)$ & $2.70(1.38,5.28)$ \\
CKD \& hypertension $(n=3817)$ & $2.58(2.00,3.34)$ \\
Atrial fibrillation \& hypertension $(n=1052)$ & $2.44(1.50,3.96)$ \\
Stroke \& hypertension $(n=2236)$ & $2.16(1.55,3.02)$ \\
Myocardial infarction \& hypertension $(n=3763)$ & $1.98(1.51,2.59)$ \\
Diabetes \& hypertension $(n=9025)$ & $1.92(1.59,2.33)$ \\
Myocardial infarction \& angina $(n=2814)$ & $1.78(1.30,2.43)$ \\
Angina \& hypertension $(n=5802)$ & $1.66(1.31,2.11)$ \\
Cancer \& hypertension $(n=7783)$ & $1.58(1.24,2.01)$ \\
Asthma \& hypertension $(n=11,242)$ & $1.29(1.03,1.60)$
\end{tabular}

Reference is those without the stated two co-occurring conditions (i.e. with asthma and hypertension vs. without asthma and hypertension [reference]) Most common co-occurring multimorbidity conditions illustrated in Fig. 1

Models adjusted for age at test, sex, ethnicity, deprivation, smoking status, body mass index, air pollution, 25-hydroxyvitamin $\mathrm{D}$, cardiorespiratory fitness, C-reactive protein, season at blood draw, and regular intake of vitamin D supplement

OR odds ratio, Clconfidence interval, $C K D$ chronic kidney disease, CPD chronic obstructive pulmonary disease

hospitalisation or death during the pandemic, further details of adverse COVID-19 outcomes such as length of hospital stay, intensive care admission, or mechanical ventilation, could provide further information. Ten potential effect modifiers were selected [4], although there are other factors which could possibly modify the association between multimorbidity and risk of severe SARS-CoV-2 infection but were unaccounted for, such as household size, occupation, and understanding and behaviour for social distancing, shielding, wearing facemasks and hand hygiene. Finally, this was an observational study, which limits our ability to establish causality.

\section{Conclusion}

In conclusion, having a new multimorbidity COVID19 index that may help identify subjects at higher risk of complication in clinical practice could be beneficial. Where stratifying the risk according to the presence of multimorbidity, and reinforcing initiatives and resources to reduce the risk in this vulnerable group of individuals should be considered as priorities in the current coronavirus pandemic.

\begin{abstract}
Abbreviations
BMI: Body mass index; Cl: Confidence intervals; CKD: Chronic kidney disease: COPD: Chronic obstructive pulmonary disease; CRP: C-reactive protein; eGFR: Estimated Glomerular Filtration Rate; IQR: Interquartile range; NHS: National Health Service; OR: Odds ratio; RR: Relative risk; UK: United Kingdom; WHO: World Health Organization; 25(OH)D: 25-Hydroxyvitamin D.
\end{abstract}

\section{Supplementary Information}

The online version contains supplementary material available at https://doi. org/10.1186/s12879-021-06600-y.

Additional file 1: Figure S1. Flow chart of participants included in the UK Biobank Study. Table S1. Literature search on the most common pre-existing comorbidities in patients with severe SARS-CoV-2 infection. Table S2. Association between multimorbidity index using 3 or more conditions and risk of severe SARS-CoV-2 infection. Table S3. Sensitivity analyses using vitamin $\mathrm{D}$ levels at follow-up and last recorded air pollution levels. Table S4. Sensitivity analyses considering time in the study and removing cardiorespiratory fitness and C-reactive protein from the model. Checklist S1. Strengthening the Reporting of Observational Studies in Epidemiology (STROBE).

\section{Acknowledgements}

This research has been conducted using the UK Biobank Resource (Reference 36371). We acknowledge the support from the National Institute for Health Research (NIHR) Applied Research Collaboration East Midlands (ARC EM), and the NIHR Leicester Biomedical Research Centre. NGF acknowledges funding from the MRC Epidemiology Unit core support (MC_UU_12015/5), and NIHR Biomedical Research Centre Cambridge: Nutrition, Diet, and Lifestyle Research Theme (IS-BRC-1215-20014). The views expressed are those of the author(s) and not necessarily those of the NIHR or the Department of Health and Social Care.

\section{Authors' contributions}

Concept and Design: NF, KK, YC. Statistical analysis: YC. Statistical support: NF, FZ, CG. Acquisition, analysis, or interpretation of data: NF, KK, FZ, CG, CR, TY, $D K, A R, M D, N I, S S$. Drafting of the manuscript: YC. Critical revision of the manuscript for important intellectual content: NF, KK, FZ, CG, CR, TY, DK, AR, MD, NI, SS. All authors agree to publish the article. All authors read and approved the final manuscript.

\section{Funding}

This research was funded by the National Institute for Health Research (NIHR) Applied Research Collaboration East Midlands (ARC EM), and the NIHR Leicester Biomedical Research Centre, and a grant from the UKRI-DHSC COVID-19 Rapid Response Rolling Call (MR/N020536/1). NGF acknowledges funding from the MRC Epidemiology Unit core support (MC_UU_12015/5), and NIHR Biomedical Research Centre Cambridge: Nutrition, Diet, and Lifestyle Research Theme (IS-BRC-1215-20014).The funders had no role in the design and conduct of the study; collection, management, analysis, and interpretation of the data; preparation, review, or approval of the manuscript; and decision to submit the manuscript for publication.

\section{Availability of data and materials}

The data that support the findings of this study are available from UK Biobank project site, subject to registration and application process. Further details can be found at https://www.ukbiobank.ac.uk. Statistical codes for this study are available at GitHub yc244. 


\section{Declarations}

Ethics approval and consent to participate

All participants gave written informed consent prior data collection. UK Biobank has full ethical approval from the NHS National Research Ethics Service (16/NW/0274).

\section{Consent for publication}

Not applicable.

\section{Competing interests}

KK is chair for SAGE subgroup on ethnicity and COVID-19 and an independent member of SAGE.

\section{Author details}

${ }^{1}$ Leicester Real World Evidence Unit, Diabetes Research Centre, Leicester General Hospital, University of Leicester, Leicester, UK. ${ }^{2} \mathrm{NIHR}$ Leicester Biomedical Research Centre, Leicester Diabetes Centre, Leicester, UK. ${ }^{3}$ Clinical Trial Service Unit and Epidemiological Studies Unit (CTSU), Nuffield Department of Population Health, University of Oxford, Oxford, UK. ${ }^{4}$ Medical Research Council Epidemiology Unit, University of Cambridge School of Clinical Medicine, Cambridge, UK.

Received: 29 September 2020 Accepted: 23 August 2021 Published online: 04 September 2021

\section{References}

1. Worldometer, Coronavirus Update (Live). https://www.worldometers. info/coronavirus/. Accessed 29 Sept 2021.

2. Jorden MARS, Villarino E, Hoferka S, Patel MT, Bemis K. Evidence for limited early spread of COVID-19 within the United States. MMWR Morb Mortal Wkly Rep. 2020;69(22):680.

3. Lipsitch M, Swerdlow DL, Finelli L. Defining the epidemiology of Covid19—studies needed. N Engl J Med. 2020;382(13):1194-6.

4. South Asian Health Foundation: COVID-19 in Black, Asian and Minority Ethnic populations: an evidence review and recommendations from the South Asian Health Foundation. 2020.

5. Emami A, Javanmardi F, Pirbonyeh N, Akbari A. Prevalence of underlying diseases in hospitalized patients with COVID-19: a systematic review and meta-analysis. Arch Acad Emerg Med. 2020;8(1):e0241265.

6. Grasselli GZA, Zanella A, Antonelli M, Cabrini L, Castelli A, Cereda D. Baseline characteristics and outcomes of 1591 patients infected with SARS-CoV-2 admitted to ICUs of the Lombardy Region, Italy. JAMA. 2020;323(16):1574-81.

7. Petrilli CM JS, Yang J, Rajagopalan H, O'Donnell L, Chernyak Y, Tobin KA, Cerfolio RJ. Factors associated with hospital admission and critical illness among 5279 people with coronavirus disease 2019 in New York City: prospective cohort study. 2020. https://doi.org/10.1136/bmj.m1966.

8. Safiya Richardson JSH, Narasimhan M, Crawford JM, McGinn T, Davidson KW. Presenting characteristics, comorbidities, and outcomes among 5700 patients hospitalized with COVID-19 in the New York City area. JAMA. 2020:323(20):2052-9.

9. Atkins JL, Masoli JAH, Delgado J, Pilling LC, Kuo C-L, Kuchel GA, Melzer D. Preexisting comorbidities predicting COVID-19 and mortality in the UK Biobank Community Cohort. J Gerontol Ser A. 2020;75(11):2224-30.

10. Academy of Medical Sciences, Multimorbidity: a priority for global health research. 2018. https://acmedsci.ac.uk/file-download/82222577. Accessed 2 Aug 2021.

11. Chudasama YVGC, Appiah K, Zaccardi F, Razieh C, Davies MJ, Yates T, Khunti K. Multimorbidity and SARS-CoV-2 infection in UK Biobank | Elsevier Enhanced Reader. Diabetes Metab Syndr. 2020;14(5):775-6.

12. Sur Roy A, Joshi A. Response to "Multimorbidity and SARS-CoV-2 infection in UK biobank." Diabetes Metab Syndr. 2020;14(5):969-969.

13. Woolford SJ, D'Angelo S, Curtis EM, Parsons CM, Ward KA, Dennison EM, Patel HP, Cooper C, Harvey NC. COVID-19 and associations with frailty and multimorbidity: a prospective analysis of UK Biobank participants. Aging Clin Exp Res. 2020;32(9):1897-905
14. Iaccarino G, Grassi G, Borghi C, Ferri C, Salvetti M, Volpe M, Investigators S-R. Age and multimorbidity predict death among COVID-19 patients: results of the SARS-RAS Study of the Italian Society of Hypertension. Hypertension. 2020;76(2):366-72.

15. McQueenie R, Foster HME, Jani BD, Katikireddi SV, Sattar N, Pell JP, Ho FK Niedzwiedz CL, Hastie CE, Anderson J, et al. Multimorbidity, polypharmacy, and COVID-19 infection within the UK Biobank cohort. PLOS ONE. 2020;15(8):e0238091.

16. Singh $A K$, Gillies $C L$, Singh $R$, Singh $A$, Chudasama $Y$, Coles $B$, Seidu S, Zaccardi F, Davies MJ, Khunti K. Prevalence of co-morbidities and their association with mortality in patients with COVID-19: a systematic review and meta-analysis. Diabetes Obes Metab. 2020. https://doi.org/10.1111/ dom.14124.

17. UK Biobank. UK Biobank: protocol for a large-scale prospective epidemiological resource. https://www.ukbiobank.ac.uk/media/gnkeyh2q/studyrationale.pdf. Accessed 2 Aug 2021.

18. UK Biobank. UK Biobank ethics and governance framework. https://www. ukbiobank.ac.uk/media/0xsbmfmw/egf.pdf. Accessed 2 Aug 2021.

19. Armstrong J RJ, Allen N, Crook D, Wilson D, Wyllie D, O'Connell AM. Dynamic linkage of COVID-19 test results between Public Health England's Second Generation Surveillance System and UK Biobank. Microb Genom. 2020;6(7):mgen000397. https://doi.org/10.1099/mgen.0.000397.

20. Arentz MYE, Klaff L, Lokhandwala S, Riedo FX, Chong M, Lee M. Characteristics and outcomes of 21 critically ill patients with COVID-19 in Washington State. JAMA. 2020;323(16):1612-4

21. Du R-H, Liang L-R, Yang C-Q, et al. Predictors of mortality for patients with COVID-19 pneumonia caused by SARS-CoV-2: a prospective cohort study. Eur Respir J. 2020;55(5):2000524. https://doi.org/10.1183/13993003. 00524-2020.

22. Guan W-j, Liang W-h, Zhao Y, et al. Comorbidity and its impact on 1590 patients with COVID-19 in China: a nationwide analysis. Eur Res J 2020;55(5):2000547. https://doi.org/10.1183/13993003.00547-2020.

23. Ji W, Huh K, Kang M, Hong J, Bae GH, Lee R, Na Y, Choi H, Gong SY, Choi $\mathrm{YH}, \mathrm{Ko} \mathrm{KP}, \mathrm{Im}$ JS, Jung J. Effect of underlying comorbidities on the infection and severity of COVID-19 in Korea: a nationwide case-control study. J Korean Med Sci. 2020;35(25):e237. https://doi.org/10.3346/jkms.2020.35. e237.

24. Li XXS, Yu M, Wang K, Tao Y, Zhou Y. Risk factors for severity and mortality in adult COVID-19 inpatients in Wuhan | Elsevier Enhanced Reader. J Allergy Clin Immunol. 2020;146(1):110-8.

25. Myers LC, The Permanente Medical Group KPNC, Oakland, Parodi SM, The Permanente Medical Group KPNC, Oakland, Escobar GJ, The Permanente Medical Group KPNC, Oakland, Liu VX, The Permanente Medical Group KPNC, Oakland. Characteristics of hospitalized adults with COVID-19 in an Integrated Health Care System in California. JAMA. 2020;323(21):2195-8.

26. Yao Q, Wang P, Wang X, Qie G, Meng M, Tong X, Bai X, Ding M, Liu W, Liu $K$, et al. A retrospective study of risk factors for severe acute respiratory syndrome coronavirus 2 infections in hospitalized adult patients. Pol Arch Intern Med. 2020;130(5):390-9.

27. Yang J, Zheng Y, Gou X, Pu K, Chen Z, Guo Q, Ji R, Wang H, Wang Y, Zhou $Y$. Prevalence of comorbidities and its effects in coronavirus disease 2019 patients: a systematic review and meta-analysis. Int J Infect Dis. 2020:94:91-5.

28. Yang X, Yu Y, Xu J, Shu H, Xia J, Liu H, Wu Y, Zhang L, Yu Z, Fang M, Yu T, Wang Y, Pan S, Zou X, Yuan S, Shang Y. Clinical course and outcomes of critically ill patients with SARS-CoV-2 pneumonia in Wuhan, China: a single-centered, retrospective, observational study. Lancet Respir Med. 2020;8(5):475-81. https://doi.org/10.1016/S2213-2600(20)30079-5.

29. Zhou FYT, Du R, Fan G, Liu Y, Liu Z, et al. Clinical course and risk factors for mortality of adult inpatients with COVID-19 in Wuhan, China: a retrospective cohort study. The Lancet. 2020;395(10229):1054-62.

30. World Health Organisation. Ambient (outdoor) air pollution. 2018. https:// www.who.int/news-room/fact-sheets/detail/ambient-(outdoor)-air-quali ty-and-health. Accessed 2 Aug 2021.

31. British Nutrition Foundation, New advice on Vitamin. 2018. https://www. nutrition.org.uk/nutritioninthenews/new-reports/983-newvitamind. html?_cf_chl_jschl_tk_=ed44f3f38c4328aae4a0590dbbd1f0459a2 4ca07-1597345628-0-AX6jK_qtRoVBhgd7ZoVrGgsVW4bYr24Hv-6wRgt x1lu-17yh6l2eMj6ls-QWmD4jwP8rxhmcIDmuAKOAPdMQqew3D1KyuzxVqMBQI_DocJ0t_UkLzbJFO9IJ2gaZXZ73z2avKzbMA4KGi-3aWC2 pOQN3RbwjFmzL8CjU8m_wg6SRlj5kvV8hzaXV279BKKC-sqUBCwszXI 
uGEc-xwpvWsEomOFz-BMpi3vz1mVG5bD1CnZPXfrivX8lu_sXBuSXCXod KZcimdj53JorwnY8u1OPRHOByABSWFONm1qLoNO6EI3RP10C_7LIu3 z0i7U97A5tQzCmCHfGgGDKTFK9RRCDDmTnr9l8mhYgGvE9n9rKwgkz2 zpOD3EYIBCjACJS3utyuXV9OPHDgDQVMtulJ7w. Accessed 2 Aug 2021.

32. Yates T, Zaccardi F, Dhalwani NN, Davies MJ, Bakrania K, Celis-Morales CA, Gill JMR, Franks PW, Khunti K. Association of walking pace and handgrip strength with all-cause, cardiovascular, and cancer mortality: a UK Biobank observational study. Eur Heart J. 2017;38(43):3232-40.

33. Pearson TA, Mensah GA, Alexander RW, Anderson JL, Cannon RO, Criqui M, Fadl YY, Fortmann SP, Hong Y, Myers GL, et al. Markers of inflammation and cardiovascular disease. Circulation. 2003;107(3):499-511.

34. Yates T, Zaccardi F, Razieh C, Gillies CL, Rowlands A, Kloecker DE, Chudasama YV, Davies MJ, Khunti K. Framework to aid analysis and interpretation of ongoing COVID-19 research. Wellcome Open Res. 2020;5(208):208.

35. Zaim S, Chong JH, Sankaranarayanan V, Harky A. COVID-19 and multiorgan response. Curr Probl Cardiol. 2020;45(8):100618.
36. Ejaz H, Alsrhani A, Zafar A, Javed H, Junaid K, Abdalla AE, Abosalif KOA, Ahmed Z, Younas S. COVID-19 and comorbidities: deleterious impact on infected patients. J Infect Public Health. 2020;13(12):1833-9.

37. Chudasama YV, Gillies CL, Zaccardi F, Coles B, Davies MJ, Seidu S, Khunti K. Impact of COVID-19 on routine care for chronic diseases: a global survey of views from healthcare professionals. Diabetes Metab Syndr. 2020;14(5):965-7.

38. Swanson JM. The UK Biobank and selection bias. The Lancet. 2012;380(9837):110

\section{Publisher's Note}

Springer Nature remains neutral with regard to jurisdictional claims in published maps and institutional affiliations.
Ready to submit your research? Choose BMC and benefit from:

- fast, convenient online submission

- thorough peer review by experienced researchers in your field

- rapid publication on acceptance

- support for research data, including large and complex data types

- gold Open Access which fosters wider collaboration and increased citations

- maximum visibility for your research: over $100 \mathrm{M}$ website views per year

At BMC, research is always in progress.

Learn more biomedcentral.com/submissions 